\title{
49. On Differential Operators permutable with Lie Continuous Groups of Transformations.
}

\author{
By Kôshichi Toyoda.
}

Mathematical Institute, Tohoku Imperial University, Sendai.

(Comm. by M. FuJiwarA, M.I.A., June 12, 1937.)

1. In the present paper, we shall generalize Casimir's theorem ${ }^{1)}$ on semi-simple continuous groups, which may be stated as follows:

Let $X_{1}, X_{2}, \ldots \ldots, X_{r}$ generate a semi-simple continuous group and satisfy the law of compositions such that

$$
\left[X_{i}, X_{k}\right]=C_{i k}^{a} X_{a}, \quad(i, k=1,2, \ldots \ldots, r) .
$$

If $\left(g^{i k}\right)$ denotes the inverse matrix of the coefficient matrix $\left(C_{i \beta}^{a} C_{k a}^{\beta}\right)$ of Cartan's quadratic form

$$
\varphi(\lambda, \lambda)=C_{i \beta}^{a} C_{k a}^{\beta} \lambda^{i} \lambda^{k},
$$

then the differential operator of the second order

$$
P(X)=g^{i k} X_{i} X_{k}
$$

is permutable with every element $X_{\omega}$, that is,

$$
X_{\omega} P(X)=P(X) X_{\omega}, \quad(\omega=1,2, \ldots \ldots, r) .
$$

By means of this theorem, Profs. B. L. van der Waerden, ${ }^{2)}$ H. Casimir and Richard Brauer ${ }^{3)}$ gave the algebraic proof of Weyl's theorem ${ }^{4}$ that all reducible representations of semi-simple continuous group are completely reducible.

2. In general, we assume that an $r$-parametric continuous group $G$ of transformation is generated by $r$ infinitesimal transformations

$$
X_{\omega}=\xi_{\omega}^{k}\left(x^{1}, x^{2}, \ldots \ldots, x^{n}\right) \frac{\partial}{\partial x^{k}}, \quad(\omega=1,2, \ldots \ldots, r),
$$

where $\xi_{\omega}^{k}\left(x^{3} x^{2}, \ldots . ., x^{n}\right)$ are analytic in a neighborhood of the origin. Then, we consider the symmetric differential operators of the $\nu$-th order, defined as follows :

$$
\begin{aligned}
& P_{0}(X)=g, \quad P_{1}(X)=g^{i} X_{i}, \quad P_{2}(X)=g^{i k} X_{i} X_{k}, \\
& \text {............................... } \quad P_{\nu}(X)=g^{i k j \ldots l} X_{i} X_{k} X_{j} \ldots \ldots X_{l} \text {, }
\end{aligned}
$$

where

$$
\begin{aligned}
& g^{i k}=g^{k i}, \quad \ldots \ldots \ldots \ldots \ldots \ldots, \quad g^{i k j \ldots l}=g^{k i j \ldots l}, \\
& g^{i k j \ldots l}=g^{i j k \ldots l} \text { etc. }
\end{aligned}
$$

1) H. Casimir: Proc. Kon..Acad. Amsterdam, 34 (1931), 844.

K. Toyoda: Japanese Journal of Mathematics, 12 (1935), 17.

2) H. Casimir und B. L. van der Waerden: Math. Annalen, 111 (1935), 1.

3) R. Brauer: Math. Zeitschrift, 41 (1936), 330.

4) H. Weyl: Math. Zeitschrift, 24 (1926), 328. 
No. 6.] On Differential Operators permutable with Lie Continuous Groups.

and we form

$$
P(X)=\sum P_{\nu}(X)=\sum g^{i k \cdots l} X_{i} X_{k} \ldots \ldots X_{l} .
$$

First, we have

Theorem 1. If a symmetric polynomial $P(\Lambda)$ be an absolute invariant of the contragredient adjoint group $H^{*}$ generated by $r$ infinitesimal transformations

$$
E_{\omega}^{*}=\Lambda_{a} C_{\omega k}^{a} \frac{\partial}{\partial \Lambda_{k}}, \quad(\omega=1,2, \ldots \ldots, r),
$$

then the corresponding differential operator $P(X)$ is permutable with every element $X_{\omega}$, that is

$$
X_{\omega} P(X)=P(X) X_{\omega}, \quad(\omega=1,2, \ldots \ldots, r) .
$$

Proof. By the law of compositions

we get

$$
\left[X_{\omega}, X_{k}\right]=C_{\omega k}^{a} X_{a}=E_{\omega}^{*} X_{k}, \quad(\omega, k=1,2, \ldots \ldots, r),
$$

$$
e^{X_{\omega}} X_{k} e^{-X_{\omega}}=e^{E_{\omega}^{*}} X_{k} .
$$

Now, for the sake of simplicity, we consider a particular case where

then, we have

$$
P(X)=g^{i k} X_{i} X_{k},
$$

$$
\begin{aligned}
e^{X_{\omega}} P(X) e^{-X_{\omega}} & =g^{i k} e^{X_{\omega}} X_{i} e^{-X_{\omega}} e^{X_{\omega}} X_{k} e^{-X_{\omega}} \\
& =g^{i k} e^{E_{\omega}^{*}} X_{i} e^{E_{\omega}^{*}} X_{k} .
\end{aligned}
$$

Hence, supposing that a symmetric polynomial $P(\Lambda)$ is an absolute invariant of the contragredient adjoint group $H^{*}$, it follows that

$$
e^{X_{\omega}} P(X)=P(X) e^{X_{\omega}}, \quad(\omega=1,2, \ldots \ldots, r),
$$

that is

$$
X_{\omega} P(X)=P(X) X_{\omega}, \quad(\omega=1,2, \ldots \ldots, r) .
$$

Remark. In order that we exclude the condition of symmetry, we have to consider an invariant bilinear form $P\left(\Lambda, \Lambda^{*}\right)=g^{i k} \Lambda_{i} \Lambda_{k}^{*}$ instead of an invariant quadratic form $P(\Lambda)=g^{i k} \Lambda_{i} \Lambda_{k}$. tions

Corollary. If a complete system of linear partial differential equa-

$$
E_{\omega}^{*} F(\Lambda)=0, \quad(\omega=1,2, \ldots \ldots, r),
$$

has $s$ independent symmetric solutions $F_{1}(\Lambda), F_{2}(\Lambda), \ldots \ldots, F_{s}(\Lambda)$, then arbitrary function $\Omega\left(F_{1}(X), F_{2}(X), \ldots \ldots, F_{s}(X)\right)$ of $F_{1}(X), F_{2}(X), \ldots \ldots$, $F_{s}(X)$, is permutable with every element $X_{\omega}$.

3. Let the parameter group ${ }^{1)} G_{0}$ of $G$ be generated by $r$ infinitesimal transformations

1) K. Toyoda: Science Reports of the Tohoku Imperial University, 24 (1935), 269. 


$$
A_{\omega}=a_{\omega}^{k}\left(\lambda_{,}^{1} \lambda_{0}^{2} \ldots \ldots, \lambda^{r}\right) \frac{\partial}{\partial \lambda^{k}}, \quad(\omega=1,2, \ldots \ldots, r),
$$

where $\lambda^{k}$ denote canonical parameters and $a_{i}^{k}(0)=\delta_{i}^{k}$ is Kronecker's delta.

Then, we have

Theorem 2. In order that a symmetric differential operator

$$
P(A)=\sum_{\nu=0}^{p} P_{\nu}(A)=\sum g^{i k \ldots l} A_{i} A_{k} \ldots \ldots A_{l}
$$

is permutable with every element $A_{\omega}$, it is necessary that each symmetric polynomial $P_{\nu}(\Lambda),(\nu=0,1, \ldots \ldots, p)$ is an absolute invariant of the contragredient adjoint group $H^{*}$.

Proof. If we suppose that

we get

$$
P(A)=g^{i} A_{i}+g^{i k} A_{i} A_{k}=0,
$$

$$
g^{i} a_{i}^{\alpha}(\lambda) \frac{\partial}{\partial \lambda^{a}}+g^{i k} a_{i}^{\alpha}(\lambda) \frac{\partial a_{k}^{\beta}(\lambda)}{\partial \lambda^{\alpha}} \frac{\partial}{\partial \lambda^{\beta}}+g^{i k} a_{i}^{\alpha}(\lambda) a_{k}^{\beta}(\lambda) \frac{\partial^{2}}{\partial \lambda^{a} \partial \lambda^{\beta}}=0,
$$

whence we obtain $g^{i k}=0$ and consequently $g^{i}=0$.

Also, we have

Theorem 3. Let $P(x)$ be a differential operator permutable with every element $X_{\omega}$ and $g(X)$ be an absolute invariant of the group $G$. If $f(x)$ be a solution of the differential equation

$$
P(X) f(x)=g(x),
$$

then $f\left(e^{X_{\omega}} x\right)$ is also a solution of the same differential equation.

Proof. If $f(x)$ be a solution of the partial differential equation

then we get

$$
P(X) f(x)=g(x),
$$

$$
\begin{aligned}
P(X) f\left(e^{X_{\omega}} x\right) & =P(X) e^{X_{\omega}} f(x)=e^{X_{\omega}} P(X) f(x) \\
& =e^{X_{\omega}} g(x)=g(x) .
\end{aligned}
$$

4. Finally, we shall give another proof for Casimir's theorem, which runs as follows.

Theorem 4. If $X_{1}, X_{2}, \ldots . ., X_{r}$ generate a semi-simple continuous group and $\left(g^{i k}\right)$ be the inverse matrix of the coefficient matrix $\left(C_{i \beta}^{a} C_{k a}^{\beta}\right)$ of Cartan's quadratic form

$$
\varphi(\lambda, \lambda)=C_{i \beta}^{a} C_{k a}^{\beta} \lambda^{i} \lambda^{k},
$$

then the differential operator of the second order

$$
P(X)=g^{i k} X_{i} X_{k}
$$

is permutable with every element $X_{\omega}$.

Proof. Since Cartan's quadratic form

$$
\varphi(\lambda, \lambda)=C_{i \beta}^{a} C_{k a}^{\beta} \lambda^{i} \lambda^{k}=g_{i k} \lambda^{i} \lambda^{k}
$$


is an absolute invariant of the adjoint group ${ }^{1)} H$ generated by $r$ infinitesimal transformations

$$
E_{\omega}=-\lambda^{a} C_{\omega a}^{k} \frac{\partial}{\partial \lambda^{k}}, \quad(\omega=1,2, \ldots \ldots, r),
$$

we have

whence

$$
\begin{aligned}
E_{\omega} \varphi(\lambda, \lambda) & =\varphi\left(\lambda, E_{\omega} \lambda\right)+\varphi\left(E_{\omega} \lambda, \lambda\right) \\
& =2 \varphi\left(\lambda, E_{\omega} \lambda\right)=0
\end{aligned}
$$

$$
g_{i a} C_{\omega k}^{a}+g_{k a} C_{\omega i}^{a}=0, \quad(\omega, i, k=1,2, \ldots \ldots, r) .
$$

Therefore, we obtain

$$
g^{i a} C_{\omega a}^{k}+g^{k a} C_{\omega a}^{i}=0, \quad(\omega, i, k=1,2, \ldots \ldots, r),
$$

which shows that the symmetric quadratic form

$$
P(\Lambda)=g^{i k} \Lambda_{i} \Lambda_{k}
$$

is an absolute invariant of the contragredient adjoint group $H^{*}$, therefore by means of Theorem 1 we can prove the following

Corollary. If $X_{1}, X_{2}, \ldots \ldots, X_{r}$ generate a semi-simple continuous group, then the determinants of all matrices $\left(C_{i k}^{\omega}\right),(\omega=1,2, \ldots \ldots, r)$, vanish simultaneously.

Furthemore, we have

Theorem 5.2) In order that a continuous group $G$ contains an element other than the identical element in the central, it is necessary and sufficient that there exists a differential operator $P(X)$ of the first order which is permutable with every element $X_{\omega}$.

Proof. If a differential operator of the first order

$$
P(X)=g+g^{i} X_{i}
$$

is permutable with every element $X_{\omega}$, then we have

$$
E_{\omega}^{*} P(\Lambda)=g^{i} \Lambda_{a} C_{\omega i}^{a}=0, \quad(\omega=1,2, \ldots . ., r),
$$

which shows that $g^{i} X_{i}$ is contained in the central.

Remark. But, if $G$ is a soluble group generated by $X_{1}, X_{2}$ such that $\left[X_{1}, X_{2}\right]=X_{1}$, then $G$ has no differential operator $P(X)$ permutable with every element $X_{\omega}$.

1) K. Toyoda: Science Reports of the Tohoku Imperial University, 25 (1936), 621.

2) This theorem was remarked by Prof. Kôsaku Yosida（全國柇上数學談話會, 123 踝). 